units: the EUROBACT International Cohort Study. Intensive Care Med 2012; 38: 1930-45.

2. Kumar A, Roberts D, Wood KE, et al. Duration of hypotension before initiation of effective antimicrobial therapy is the critical determinant of survival in human septic shock. Crit Care Med 2006; 34: 1589-96.

3. Schuts EC, Hulscher MEJL, Mouton JW, et al. Current evidence on hospital antimicrobial stewardship objectives: a systematic review and meta-analysis. Lancet Infect Dis 2016; 16: 847-56.

4. Huang AM, Newton D, Kunapuli A, et al. Impact of rapid organism identification via matrix-assisted laser desorption/ionization time-of-flight combined with antimicrobial stewardship team intervention in adult patients with bacteremia and candidemia. Clin Infect Dis 2013; 57: 1237-45.

5. Sakarikou C, Altieri A, Bossa MC, et al Rapid and cost-effective identification and antimicrobial susceptibility testing in patients with Gramnegative bacteremia directly from blood-culture fluid. J Microbiol Methods 2018; 146: 7-12.

6. Ling TK, Liu ZK, Cheng AF. Evaluation of the VITEK 2 system for rapid direct identification and susceptibility testing of gram-negative bacilli from positive blood cultures. J Clin Microbiol 2003; 41: 4705-7.

7. Bruins MJ, Bloembergen P, Ruijs GJ, Wolfhagen MJ. Identification and susceptibility testing of Enterobacteriaceae and Pseudomonas aeruginosa by direct inoculation from positive BACTEC blood culture bottles into Vitek 2. J Clin Microbiol 2004; 42: 7-11.

8. Jorgensen JH. Selection criteria for an antimicrobial susceptibility testing system. J Clin Microbiol 1993; 31: 2841-4.

DOI: https://doi.org/10.1016/j.pathol.2021.03.013

\section{Weak positive SARS-CoV-2 N2 gene results using the Xpress Xpert assay: the need for an alternate interpretative criteria in a low prevalence setting}

Sir,

The Xpert Xpress SARS-CoV-2 (Xpert) test (Cepheid, USA) is the most common Australian rapid nucleic acid test (NAT) used for detection of SARS-CoV-2 in nasal and nasopharyngeal specimens. This rapid RT-PCR assay detects two targets: the E (envelope) and N2 (nucleocapsid) SARS-CoV2 coding regions. The assay cartridge is a closed system that includes sample processing, probe check internal controls which ensure adequate sample processing, and checks for sample-associated inhibition and reagents functionality. ${ }^{1}$ Following the manufacturer's instructions ${ }^{1}$ the results are interpreted automatically by the assay software into four categories: 'positive', 'presumptive positive', 'negative' and 'error'. Specimens positive for both E and N2 targets, or single N2 target, are reported as 'positive', while a single E target positive result is reported as 'presumptive positive'.

The Xpert assay is currently performed at 37 NSW Health Pathology (NSWHP) laboratories, and by 10 May 2021, 55,411 tests had been performed on nasal or nasopharyngeal swabs. A total of 317 samples tested positive for both $E$ and N2 targets. There were 42 notified instances where only N2 target positive results were obtained and two where only the E target was detected. All of these 44 tests detected the target at a high cycle threshold $(\mathrm{Ct})$ number (range 36.8-44.6, average 42.0). Of the $42 \mathrm{~N} 2$ target only positive tests, 16 $(38.1 \%)$ were deemed as true infection as confirmed by alternate NAT, SARS-CoV-2-specific serology, and/or consistent epidemiological exposure (Table 1). The remaining $26(61.9 \%)$ individuals with only N2 target positive results had neither clinical nor epidemiological clues

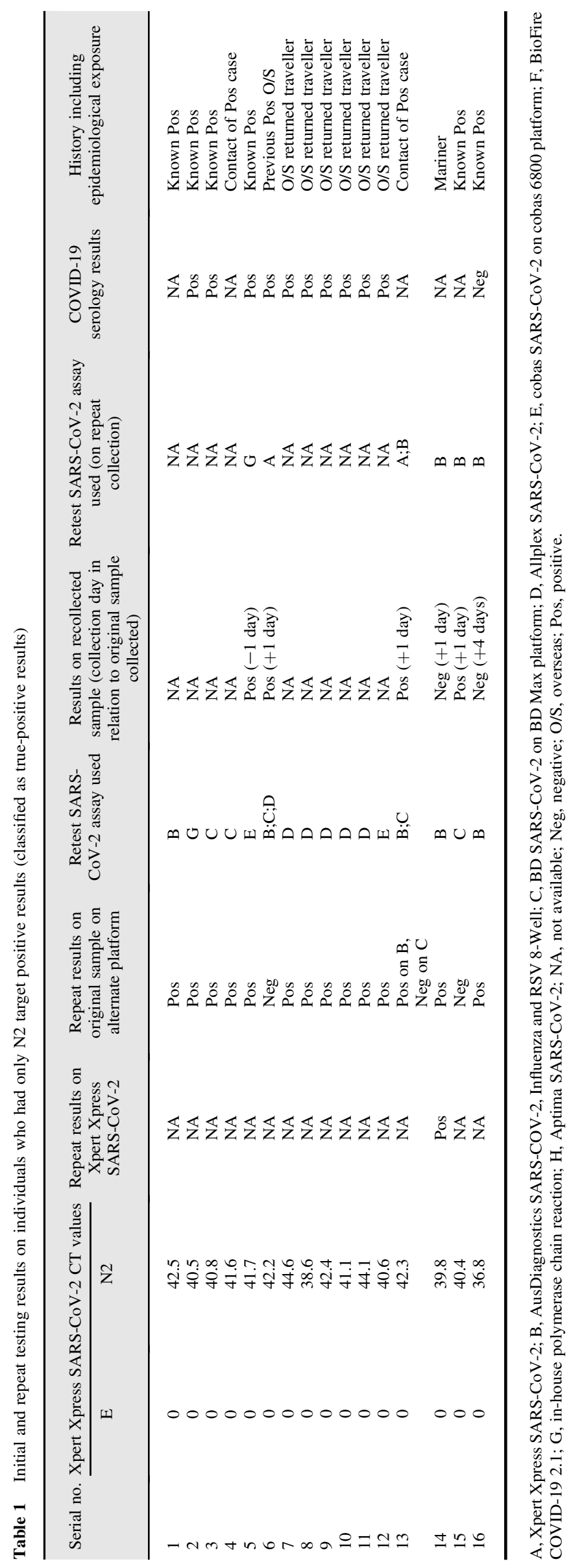


Table 2 Initial and repeat testing results on individuals who had only N2 target positive results (classified as false-positive results)

\begin{tabular}{|c|c|c|c|c|c|c|c|c|}
\hline \multirow[t]{2}{*}{ Serial no. } & \multicolumn{2}{|c|}{ Xpert Xpress SARS-CoV-2 CT values } & \multirow{2}{*}{$\begin{array}{l}\text { Repeat results on } \\
\text { Xpert Xpress } \\
\text { SARS-CoV-2 }\end{array}$} & \multirow{2}{*}{$\begin{array}{l}\text { Repeat results on } \\
\text { original sample on } \\
\text { alternate platform }\end{array}$} & \multirow{2}{*}{$\begin{array}{l}\text { Retest SARS-CoV-2 } \\
\text { assay used }\end{array}$} & \multirow{2}{*}{$\begin{array}{l}\text { Results on } \\
\text { recollected sample } \\
\text { (collection day in } \\
\text { relation to original } \\
\text { sample collected) }\end{array}$} & \multirow{2}{*}{$\begin{array}{l}\text { Retest SARS- } \\
\text { CoV-2 assay used } \\
\text { (on repeat } \\
\text { collection) }\end{array}$} & \multirow[t]{2}{*}{ COVID-19 serology results } \\
\hline & $\mathrm{E}$ & $\mathrm{N} 2$ & & & & & & \\
\hline 1 & 0 & 40.8 & NA & NA & NA & $\operatorname{Neg}(+1$ day $)$ & A & NA \\
\hline 2 & 0 & 43.4 & NA & Neg & $\mathrm{D}$ & Neg (same day) & $\mathrm{D}$ & Neg \\
\hline 3 & 0 & 42.3 & $\mathrm{Neg}$ & Neg & $\mathrm{B} ; \mathrm{G}$ & NA & NA & NA \\
\hline 4 & 0 & 43.5 & NA & Neg & G & Neg (same day) & G & Neg \\
\hline 5 & 0 & 43.5 & $\mathrm{Neg}$ & Neg & $\mathrm{B} ; \mathrm{G}$ & Neg (same day) & $\mathrm{A} ; \mathrm{B} ; \mathrm{G}$ & Neg \\
\hline 6 & 0 & 40.9 & NA & Neg & G & Neg (+1 day) & A & NA \\
\hline 7 & 0 & 42.7 & $\mathrm{Neg}$ & Neg & $\mathrm{D}$ & Neg (same day) & $\mathrm{A} ; \mathrm{D}$ & NA \\
\hline 8 & 0 & 42.0 & NA & Neg & $\mathrm{C}$ & NA & NA & NA \\
\hline 9 & 0 & 44.9 & NA & Neg & $\mathrm{D}$ & Neg (same day) & $\mathrm{A} ; \mathrm{C}$ & Neg \\
\hline 10 & 0 & 43.9 & NA & $\mathrm{Neg}$ & $\mathrm{C}$ & Neg (same day) & $\mathrm{C}$ & NA \\
\hline 11 & 0 & 44.0 & NA & NA & NA & NA & NA & NA \\
\hline 12 & 0 & 41.5 & NA & NA & NA & $\operatorname{Neg}(+1$ day $)$ & $\mathrm{A} ; \mathrm{C}$ & NA \\
\hline 13 & 0 & 42.9 & $\mathrm{Neg}$ & Neg & $\mathrm{C} ; \mathrm{D}$ & Neg (same day) & $\mathrm{A} ; \mathrm{C}$ & NA \\
\hline 14 & 0 & 42.2 & $\mathrm{Neg}$ & Neg & $\mathrm{B} ; \mathrm{C} ; \mathrm{D}$ & Neg (same day) & $\mathrm{A} ; \mathrm{C}: \mathrm{D}$ & NA \\
\hline 15 & 0 & 43.0 & NA & Neg & $\mathrm{D} ; \mathrm{H}$ & Neg (same day) & A & Neg \\
\hline 16 & 0 & 42.0 & NA & Neg & $\mathrm{D}$ & Neg (same day) & A & Neg \\
\hline 17 & 0 & 40.6 & $\mathrm{Neg}$ & Neg & $\mathrm{D}$ & NA & NA & NA \\
\hline 18 & 0 & 40.7 & $\mathrm{Neg}$ & Neg & $\mathrm{C}$ & NA & NA & Neg \\
\hline 19 & 0 & 44.5 & $\mathrm{Neg}$ & Neg & B & $\mathrm{Neg}$ (+1 day) & $\mathrm{A} ; \mathrm{B}$ & NA \\
\hline 20 & 0 & 42.8 & $\mathrm{Neg}$ & Neg & $\mathrm{C}$ & Neg (same day) & $\mathrm{A}$ & NA \\
\hline 21 & 0 & 41.1 & $\mathrm{Neg}$ & Neg & $\mathrm{C}$ & NA & NA & NA \\
\hline 22 & 0 & 42.9 & Neg & Neg & $\mathrm{C}$ & Neg (same day) & $\mathrm{C}$ & NA \\
\hline 23 & 0 & 42.6 & $\mathrm{Neg}$ & Neg & $\mathrm{H}$ & Neg (+1 day) & $\mathrm{A} ; \mathrm{D}$ & NA \\
\hline 24 & 0 & 41.7 & Neg & Neg & $\mathrm{C}$ & Neg (same day) & $\mathrm{A} ; \mathrm{C} ; \mathrm{D}$ & NA \\
\hline 25 & 0 & 42.1 & Neg & Neg & $\mathrm{C}$ & NA & NA & NA \\
\hline 26 & 0 & 41.3 & Neg & Neg & $\mathrm{B} ; \mathrm{F}$ & Neg $(+1$ day $)$ & $\mathrm{A} ; \mathrm{F}$ & Pos \\
\hline
\end{tabular}

A, Xpert Xpress SARS-CoV-2; B, AusDiagnostics SARS-COV-2, Influenza and RSV 8-Well; C, BD SARS-CoV-2 on BD Max platform; D, Allplex SARS-CoV-2; E, cobas SARS-CoV-2 on cobas 6800 platform; F, BioFire COVID-19 2.1; G, in-house polymerase chain reaction; H, Aptima SARS-CoV-2; NA, not available; Neg, negative; Pos, positive. 
suggestive of COVID-19 (Table 2), and all had $\mathrm{Ct}$ values over 40 (range 40.6-44.9, average 42.5). The test was repeated on the same sample and Xpert assay for $15 / 26$ $(57.7 \%)$ specimens and all were negative. Nineteen of the 26 $(73.1 \%) \mathrm{N} 2$ target positive individuals had a repeat specimen collected and all were negative using the Xpert assay and/or alternate SARS-CoV-2 NAT. SARS-CoV-2-specific serology was performed in $8 / 26(30.8 \%)$ with one patient returning a positive result (SARS-CoV-2-specific IgG at a titre of 640 using a reference SARS-CoV-2 immunofluorescence assay $^{2}$ and Xpress Xpert N2 target positive at Ct 41.3 collected in May 2021). This patient was a known COVID-19 NAT positive case from March 2020 and had received their first dose of the AstraZeneca COVID-19 vaccine a few weeks prior to the Xpert test, a possible explanation of the positive SARS-CoV-2-specific IgG.

What do single target $\mathrm{N} 2$ results mean? In a high prevalence setting (where the assay was developed) a single target positive result may indicate early infection, late infection, or reflect a false-positive finding. One USA study published in October 2020 where there was a high prevalence of COVID19 reported 4\% E negative N2 positive results among 1123 positive Xpert results. ${ }^{3}$ Approximately one-third (29.5\%) were symptomatic at clinical presentation, and they concluded that the late single $\mathrm{N} 2$ positive results suggested prolonged subclinical or resolving infections. An Italian study reported that all 12/1639 samples tested with N2 only positive results with a $\mathrm{Ct}>39$ were established to be false-

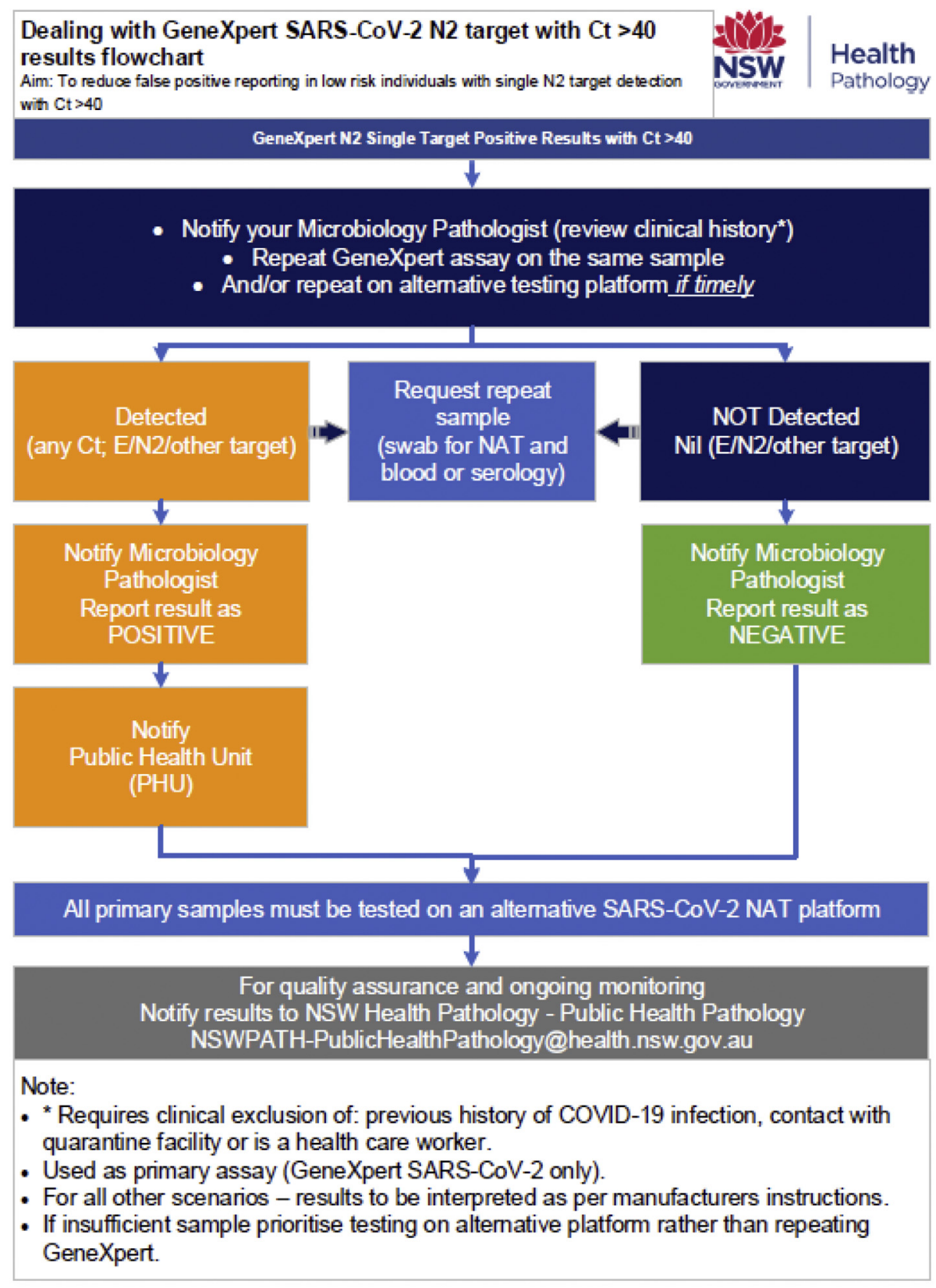

300421 Approved by NSWHP IMT CO VID-19 Pandemic Response Prepared by NSWHP-Public Health Pathology

Fig. 1 Suggested flowchart developed to deal with isolated N2 target positive results with CT $>40$ on the Xpert assay in a low prevalence setting. 
positives. ${ }^{4}$ In this study, repeat testing on the same or alternative platform was performed after a centrifugation step. It should be noted that the manufacturer does not recommend repeat testing for $\mathrm{E}$ negative $\mathrm{N} 2$ positive results, regardless of $\mathrm{Ct}$ values, and instructs these samples to be reported as 'positive'. In our low prevalence setting in Australia, all N2 only positive samples with a $\mathrm{Ct}$ greater than 40 were found to be false-positives once a clinical history had been obtained excluding prior COVID-19 infection or exposure to high-risk settings (either contact with a known COVID-19 case or residing in a hotel quarantine facility). These findings were supported by further testing on the same or a freshly collected sample. The samples in our study that were deemed to be 'true-positive' findings were generally observed during the initial stages of the pandemic of 2020 when disease prevalence was higher.

Factors such as the site and quality of sampling, swab type and viral transport media, timing of collection, and sensitivity of the platforms employed need to be considered when interpreting single target high $\mathrm{Ct}$ positive results. An interlaboratory quality assurance survey performed across all NSWHP laboratories in November 2020 revealed no major differences in Xpert $\mathrm{Ct}$ values across a range of different viral transport media at a concentration of as low as $10^{3}$ copies per $\mathrm{mL}$ (NSWHP SARS-CoV-2 Internal Quality Assurance Survey; data not shown). It has been reported that prolonged virus shedding is observed in lower respiratory tract compared to upper respiratory tract specimens (median duration 34 vs 19 days post-infection) ${ }^{5}$ and whether the former might be considered to confirm the initial positive results needs assessment. A study reported increased analytical sensitivity of the Xpert assay over the BD Max assay (BD Diagnostics, Canada) which could explain the variation in the results on the same specimen across NAT platforms. ${ }^{6}$ One of the limitations in the study was that two of 26 recollected specimens were tested solely by BD Max. The differential performance in sensitivity across platforms could have resulted in a 'false-negative' result. While it is plausible that the E negative, N2 positive results in our setting may have represented early infection before other markers became available, the absence or progression of transmission events and lack of clusters associated with the index case affirms a 'false-positive' finding.

The evaluations of many 'Emergency Use Only' authorised NAT were mostly based on the analytical performance in terms of sensitivity and specificity: they lacked data on actual diagnostic performance based on prospective clinical evaluation due to early urgent deployment of these assays during the initial pandemic stages. This resulted in limited knowledge of predictive values prior to laboratory adoption of assays. With the low incidence of COVID-19 in Australia (less than $1 \%$ daily positivity rate as at 31 May 2021, www. health.gov.au), one should expect a lower positive predictive value (including highly specific assays) and a two-step testing algorithm may be required to resolve the 'weak' positive results. These weak positive $(\mathrm{Ct}>40)$, E negative, $\mathrm{N} 2$ only positive results pose several challenges for contact tracers in that the initial 'positive' finding triggers significant public health measures. This is especially true when these results stem from smaller laboratories that have no alternate testing platforms available for immediate confirmation. We calculated that the confirmatory steps such as repeat specimen collection and re-testing could result in an additional cost.
The financial and social impact costs of triggering COVID-19 testing in a region, on the basis of a later confirmed falsepositive result, cannot be underestimated. There is an urgent need for a standardised reporting pathway for such 'weak' positive single target results. A study in a low endemic setting suggests that isolated N2 positive results with a $\mathrm{Ct}$ value of $>40$ can be reported as 'not detected'. ${ }^{7} \mathrm{We}$ suggest a flowchart (Fig. 1) that may assist with initial interpretation and further confirmation of weak positive N2 results. This flowchart is contingent upon a low prevalence environment, appropriate clinical history taking, and retesting and re-sampling based on the clinical scenario, and should be reviewed as the situation changes.

To conclude, we report 42 cases of E negative, N2 positive results on the Cepheid rapid Xpert Xpress SARS-CoV-2 assay. In this study we found that if the flowchart of Fig. 1 is used along with appropriate clinical and epidemiological history, no false-positive results would have been reported. To our knowledge, this is the first report from Australia and a large-scale study from a low prevalence setting. Given the downstream public health and clinical implications, we suggest a flowchart that laboratories in a low prevalence setting might consider when reporting such results. Interpretation of such results requires caution and a close liaison with the medical microbiologist and public health physician to ensure an accurate clinical history is obtained.

Acknowledgements: The authors acknowledge the following NSWHP laboratories that contributed data to this study: Armidale, Bankstown, Blacktown, Broken Hill, Campbelltown, Canterbury, Coffs Harbour, Gosford, Griffith, John Hunter Hospital, Lismore, Liverpool, Nepean, Orange, Randwick, Royal Prince Alfred Hospital, St George, Tamworth, Tweed Heads, ICPMR Westmead, Wollongong, and the Pandemic Incident Management Team who have been managing the NSWHP COVID-19 response.

Conflicts of interest and sources of funding: The authors state that there are no conflicts of interest to disclose.

Hemalatha Varadhan, Vishal Ahuja, Catherine Pitman, Dominic E. Dwyer, on behalf of NSW Health Pathology

\section{NSW Health Pathology, NSW, Australia}

Contact Dr Hemalatha Varadhan.

E-mail: hemalatha.varadhan@health.nsw.gov.au

1. Cepheid GeneXpert. Xpert Xpress SARS-CoV-2: Instructions for Use. Sunnyvale, CA: Cepheid, 2020. https://www.cepheid.com/Package\% 20Insert $\%$ 20Files/Xpert\%20Xpress \%20SARS-CoV-2\%20Assay\% 20ENGLISH\%20Package\%20Insert\%20302-3787\%20Rev.\%20B.pdf

2. Hueston L, Kok J, Guibone A, et al. The antibody response to SARSCoV-2 infection. Open Forum Infect Dis 2020; 7: https://doi.org/ 10.1093/ofid/ofaa387.

3. Khoshchehreh M, Wald-Dickler N, Holtom P, Butler-Wu SM. A needle in the haystack? Assessing the significance of envelope (E) genenegative, nucleocapsid (N2) gene-positive SARS-CoV-2 detection by the Cepheid Xpert Xpress SARS-COV-2 assay. J Clin Virol 2020; 133: 104683.

4. Falasca F, Sciandra I, Di Carlo D, et al. Detection of SARS-COV N2 gene: very low amounts of viral RNA or false positive? J Clin Virol 2020; 133: 104660 .

5. Murphy K. SARS CoV-2 detection from upper and lower respiratory tract specimens: diagnostic and infection control implications. Chest 2020; 158: $1804-5$. 
6. Mostafa HH, Hardick J, Morehead E, Miller JA, Gaydos CA, Manabe YC. Comparison of the analytical sensitivity of seven commonly used commercial SARS-CoV-2 automated molecular assays. J Clin Virol 2020; 130: 104578 .

7. Rakotosamimanana N, Randrianirina F, Randremanana R, et al. GeneXpert for the diagnosis of COVID-19 in LMICs. Lancet Glob Health 2020; 8: e1457-8.

DOI: https://doi.org/10.1016/j.pathol.2021.10.001

\section{Analytical sensitivity and specificity of the Cepheid Xpert Xpress SARS-CoV-2/FIu/RSV assay}

Sir,

The COVID-19 pandemic caused by SARS-CoV-2 is a public health emergency on a global scale, with over 85 million cases worldwide as of 6 January 2021. Along with clear and decisive public health interventions, one of the cornerstones in controlling the pandemic is rapid and accurate diagnostic testing, with reverse transcription polymerase chain reaction (RT-PCR) testing considered the 'gold standard' testing method. ${ }^{2}$ The clinical spectrum of COVID-19 is broad, with an overlap between COVID-19 clinical features and symptoms of other common respiratory viral infections such as influenza and respiratory syncytial virus (RSV). ${ }^{3}$ Given the importance of early detection of cases of COVID-19, rapid discrimination between SARS-CoV-2 and other respiratory viruses is essential.

The Xpert Xpress SARS-CoV-2 assay (Cepheid, USA) has been used in many countries for the rapid detection of SARSCoV-2, with high sensitivity and specificity, ${ }^{4,5}$ including 89 remote point of care testing sites across Australia. ${ }^{6}$ The assay detects both the pan-sarbecovirus $\mathrm{E}$ gene and the $\mathrm{N} 2$ region of the $\mathrm{N}$ gene specific to SARS-CoV-2 in approximately 45 minutes. ${ }^{4}$ Recently, the Cepheid Xpert Xpress SARS-CoV-2/ Flu/RSV assay has received Emergency Use Authorization (EUA) from the United States Food and Drug Administration (FDA). ${ }^{7}$ It is designed to detect and differentiate SARS-CoV2 , influenza A, influenza B and RSV in nasopharyngeal swabs, nasal swabs or nasal washes/aspirates and is used on GeneXpert Systems. Analytical results are available within 36 minutes and provide a single detected or not detected result for each virus type and a matching $\mathrm{Ct}$ value result for interpretation.

Here, we undertook a clinical and laboratory validation study to evaluate the analytical sensitivity and specificity of the Xpert Xpress SARS-CoV-2/Flu/RSV assay.

Testing was conducted at the Microbiological Diagnostic Unit Public Health Laboratory (MDU PHL), The University of Melbourne at the Doherty Institute, Melbourne, Australia. In brief, SARS-CoV-2-positive nasopharyngeal or deep nasal swabs were obtained from routine clinical testing at MDU PHL, and stored SARS-CoV-2-negative nasopharyngeal or deep nasal swabs were obtained from the Department of Microbiology, Royal Melbourne Hospital (RMH), Melbourne, Australia.

All clinical samples were previously tested for SARSCoV-2 using the AusDiagnostics Coronavirus Typing (8well) panel (AusDiagnostics, Australia) at RMH, as previously described, ${ }^{8}$ and using the Aptima SARS-CoV-2 assay
(Hologic, USA) at MDU PHL, according to the manufacturer's instructions.

Analytical sensitivity for SARS-CoV-2 detection was assessed using two approaches. First, a $50 \mu \mathrm{L}$ volume of quantified inactivated whole virus (SARS-CoV-2 Analytical Q Panel; Qnostics, UK) supplied as a standardised dilution series $(6.0-1.7 \log 10$ digital copies/mL) was spiked into universal transport media (UTM). Subsequently, $300 \mu \mathrm{L}$ of spiked UTM was used in the Xpert Xpress SARS-CoV-2/Flu/RSV assay and also in the Xpert Xpress SARS-CoV-2 assay for comparison. The limit of detection (LOD) was determined, and all testing was performed in triplicate. Second, analytical sensitivity was also determined using heat-killed SARS-CoV-2 virus stock quantified at $1.04 \times 10^{5} \mathrm{TCID}_{50} / \mathrm{mL}$, obtained from previously isolated SARS-CoV-2 in Melbourne.? Virus was diluted in saline, and $50 \mu \mathrm{L}$ was spiked into universal transport media (obtained from the Media Preparation Unit, University of Melbourne). Three replicates at $10 \times, 1 \times$ and $0.1 \times$ LOD (LOD determined by Xpert Xpress SARS-CoV2 ) were tested. Clinical sensitivity was assessed by testing 46 RT-PCR confirmed positive samples (previously tested on the Panther Fusion SARS-CoV-2 assay; Hologic), spanning a range of cycle $\mathrm{Ct}$ values between 18.2 and 36.1 (Table 1).

Cross-reactivity was assessed using a commercial panel of respiratory control organisms (NATRPC2-BIO; Zeptometrix, USA) comprising purified, intact virus particles and bacterial cells suspended in a matrix that mimics the composition of a clinical specimen. Cross-reactivity was also examined using gamma-irradiated influenza virus (A/Victoria/31/2020 and B/ Darwin/58/2019), respiratory syncytial virus (RSV) A 16144363 and RSV B 15136810 and two seasonal human coronavirus strains OC43 and 229E obtained from the Victorian Infectious Diseases Reference Laboratory (VIDRL), Melbourne, Australia, and spiked into pooled nasopharyngeal swab samples that tested negative to SARSCoV-2. Clinical specificity was assessed by testing 50 SARSCoV-2 RT-PCR-negative samples obtained from patients with respiratory symptoms attending $\mathrm{RMH}$.

Using the Qnostics SARS-CoV-2 panel, the LoD for SARS-CoV-2 with the Xpert Xpress SARS-CoV-2/Flu/RSV assay was 8.3 copies $/ \mathrm{mL}$, and for the Xpert Xpress SARSCoV-2, assay was 8.3 copies $/ \mathrm{mL}$. Using dilutions of heat inactivated SARS-CoV-2, the LoD for the Xpert Xpress SARS-CoV-2/Flu/RSV assay was 0.002 TCID $_{50} / \mathrm{mL}$, and for the Xpert Xpress, SARS-CoV-2 assay was 0.002 TCID $_{50}$ /

Table 1 Sensitivity and specificity of the Xpert Xpress SARS-CoV-2/Flu/ RSV test with the comparator SARS-CoV-2 assay (using the Aptima SARSCoV-2 assay)

\begin{tabular}{lccc}
\hline GeneXpert Ct & GeneXpert $^{\mathrm{a}}$ & RT-PCR $^{\mathrm{b}}$ & Concordance (\%) $^{\text {a }}$ \\
\hline Positive samples & & & \\
$\quad<20$ & 21 & 21 & 100 \\
$20-25$ & 10 & 10 & 100 \\
$26-30$ & 9 & 6 & 100 \\
$>30$ & 6 & 50 & 100 \\
Negative samples & 50 & & 100 \\
$\quad$ N/A & &
\end{tabular}

${ }^{\mathrm{a}}$ Tested on the Xpert Xpress SARS-CoV-2/Flu/RSV assay.

${ }^{\mathrm{b}}$ Tested on the Aptima SARS-CoV-2 assay. 